\title{
Maxillofacial infections of odontogenic origin : Odontopathogens and antibiotic sensitivity : A demographic cross-sectional study in Elsharqia Governorate
}

\section{Original Article}

\author{
Ahmed M. Habib, Nader Elbokle, Maha Hakam \\ Department of Oral and Maxillofacial Surgery, Faculty of Oral and Dental Medicine, Cairo \\ University, Cairo, Egypt
}

\begin{abstract}
Aim: : To assess the most common micro-organisms causing odontogenic infections and the most effective antibiotic against.

Materials and Methods: The study was conducted on 150 patients with maxillofacial infection. The pus sample was collected, cultured (aerobically and anaerobically) and stained for morphological study of the isolates. Antibiotic sensitivity test for the isolates were performed.

Results: A total of 260 micro-organisms were isolated, Pure aerobes were identified in 54(36\%) of cases, pure anaerobes in $8(5 \%)$, mixed aerobes and anaerobes in $79(53 \%)$ andno pathogenic organism were isolated in $9(6 \%)$. Among the entire aerobic isolates, Ciprofloxacin and Amoxicillin/clavulanic acid were the most effective drug (100\%) followed by Clindamycin (90\%). The leasteffective drugs were amoxicillin (85\%). Among the entire anaerobic isolates, Metronidazole was the most effective drug (100\%) followed by Ciprofloxacin, Amoxicillin/clavulanic acid, Clindamycin (90\%) each and Cefotaxime(80\%). The leasteffective drug was amoxicillin (100\%).

Conclusion: The most common bacteria isolated were Staphylococcus spp., Streptococcus spp., Klebsiella spp., Prevotella spp., Peptostreptococcus spp. Ciprofloxacin, Amoxicillin/clavulanic acid and Clindamycin were the most effective drugs for all isolates. The least effective drug was amoxicillin.
\end{abstract}

Key Words: Antibiotic sensitivity, dentoalveolar abscess, maxillofacialodontogenic infection.

Received: 15 March 2019, Accepted: 31 May 2019

Corresponding Author: Ahmed Mohamed Habib, Department of Oral and Maxillofacial Surgery, Faculty of Oral and Dental Medicine, Cairo University, Tel.: +201007228382, E-mail: dr.eldok@yahoo.com.

ISSN: 2090-097X, January 2019, Vol. 10, No. 1

\section{INTRODUCTION}

Human body normally contains hundreds of different species of bacteria. Most of these bacteria present on the skin, the mucous membranes and in the spaces between the teeth and the gums. Every different site of the body represents a different environment that is why it will possess a different type of bacteria.

In dental abscess, once the bacteria reach the pulp chamber, bacteria start to colonize the root canal system forming biofilm. Shortly after root canals colonization, bacteria progresses to the periapical tissues through the apical foramen. Once it reaches the periapical tissues, these bacteria start inducing acute inflammation that ends with pus formation. If these dental infections were not treated as early as possible, it could spread to the nearby fascial spaces and important anatomic structures giving rise to serious complications such as septicemia, cavernous sinus thrombosis, brain abscess, shock, and eventually death.

The present study was designed based on the importance of odontogenic infection and its serious complications, geographic differences in the abscess microbiota, lack of sufficient evidence to support the use of one antibiotic regimen over another or to indicate one treatment modality over another. In addition, the great concern about antibiotic resistance, and scarcity of such studies concerning that in Egypt.

\section{MATERIALS AND METHODS}

\section{Patient Selection:}

A total of one hundred and fifty consecutive patients diagnosed for having oral and maxillofacial infections 
of odontogenic origin were selected from the Dental Department at Belbeis General Hospital and Bordeen Integrated Hospital in El-sharqia.

\section{Inclusion Criteria:}

1. Patients having isolated or multiple maxillofacial infections that assessed radiographically using periapical or panoramic views.

2. Patients showing swelling intra-oral and/or extraoral, fistula, redness, trismus, and lymphadenopathy.

3. Patient of all age groups and gender.

\section{Exclusion Criteria:}

Patients already on antibiotics, full preoperative assessment including medical, dental history, clinical examination and radiographic examination was completed for every patient. The gathered information concerning history taking and physical examination was recorded into diagnostic sheets.

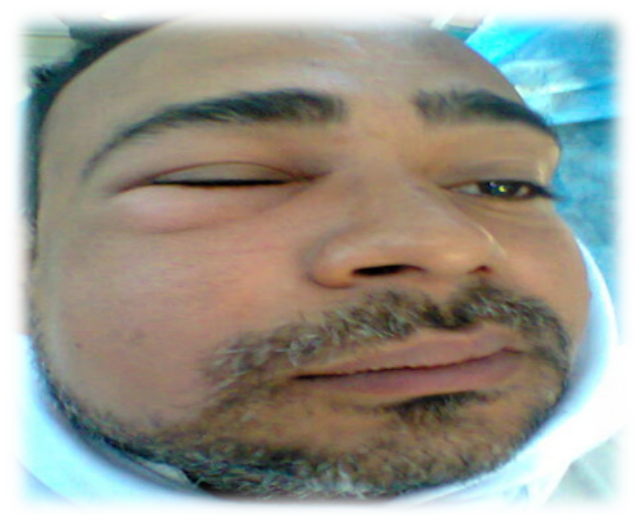

Fig. 1: extra-oral photograph showing canine and buccal space involvement

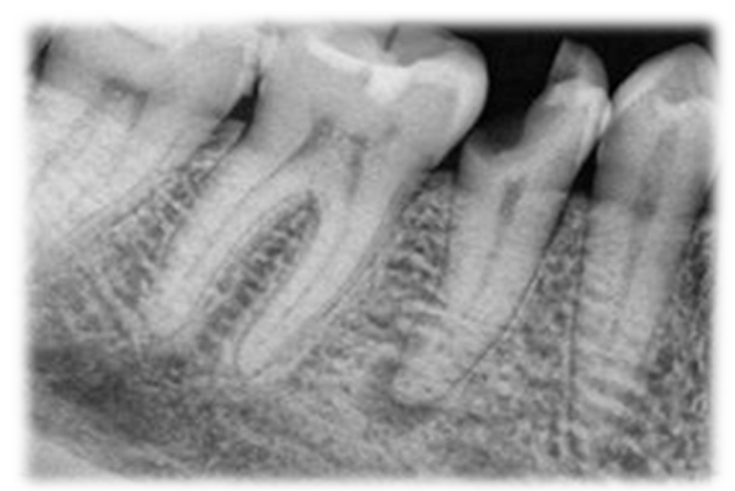

Fig. 2: badly decayed mandibular second premolar with periapical radiolucency around the root apex
Specimens were collected throughthe mucous membrane or the skin covering the affected area were surgically scrubbed by povidone-iodine $10 \%$ and dried. The specimens were taken either by 20 cubic centimeter disposable plastic syringe as a standard (fig.3) or by sterile swab in cases where there is a discharging sinus tract. Air was carefully expelled from the syringe after taking the specimen, then its top was recapped, and the aspirate was sent to the microbiological laboratory within 2 hours to be processed. The swab was immediately inoculated into a tube of thioglycollate broth.

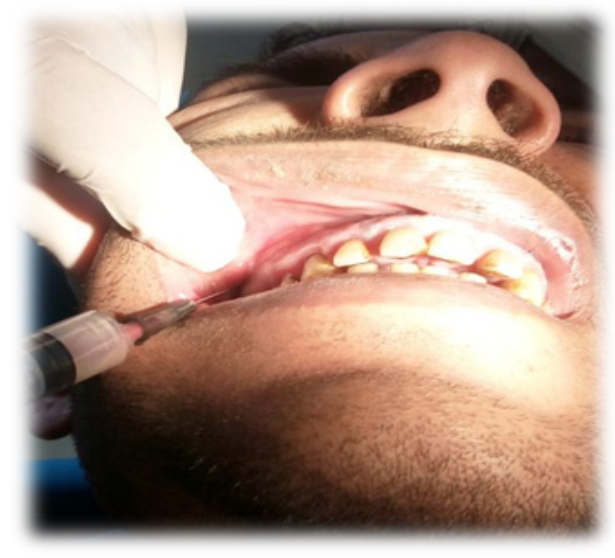

Fig. 3: Aspiration of the pus specimen

The specimens were incubated for 24 hours at $37 \mathrm{o} \mathrm{c}$. then subcultured onto 2 solid agar plates, one blood agar plate for aerobic incubation for 24 hours and one brain heart infusion agar for anaerobic incubation for 48-72 hours. All the anaerobic and aerobic plates were examined. The colonies of bacteria were identified by their macroscopic and microscopic appearance. (Fig. 4) Biochemical tests (catalase, oxidase, coagulase, indole test) were performed. Antimicrobial susceptibility testing was performed for aerobic and anaerobic isolates, by disc diffusion method. (Fig. 5) The following antibiotics were selected for testing:Amoxicillin, Amoxicillin\&clavulanic acid, Clindamycin, Cefotaxime, Metronidazole, Ciprofloxacin.

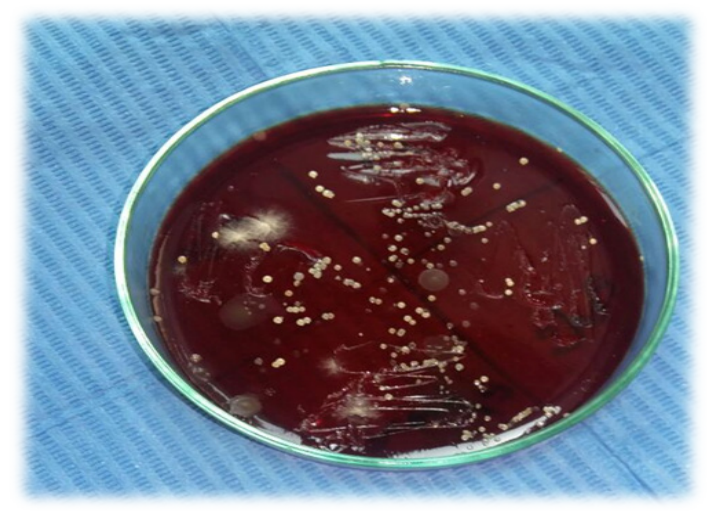

Fig. 4: Bacterial colonies on blood agar plate 


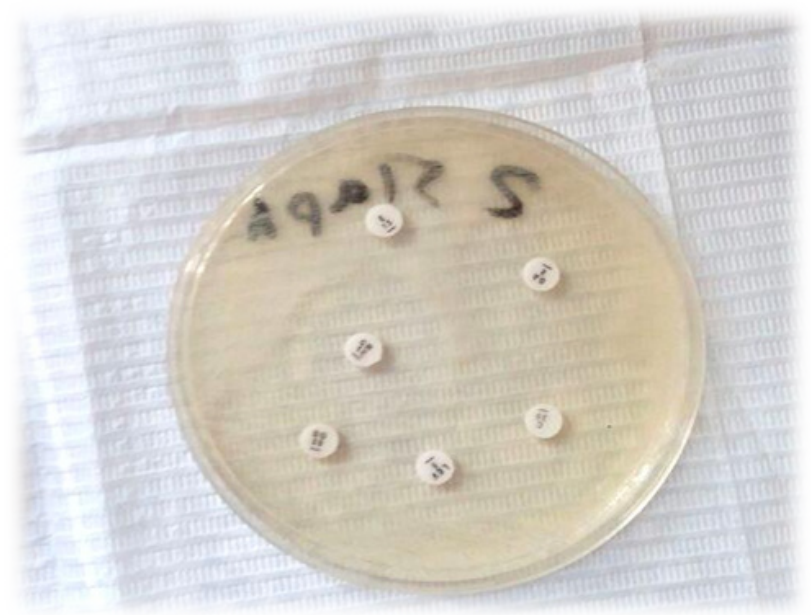

Fig. 5: Discs of the antibiotic sensitivity test

All patients included in the present study were treated by combination of medical treatment (in form of preoperative broad spectrum antibiotic) and surgical treatment (in form of incision and drainage with extraction of the offending tooth).

\section{RESULTS}

I- Demographic and clinical data: A- Demographic data:

150 patients with maxillofacial infections of the odontogenic origin participated in this study. They were selected from the dental Department at Belbeis General Hospital and Bordeen Integrated Hospital in El-sharqia,the mean age of patients was $30.0 \pm 15.5$ years and ranged from 5 to 65 years, $47.3 \%$ of them were females and $52.7 \%$ were males.

\section{B-Clinical presentation:}

Patients may present by more than one symptom and sign. Erythema was present in most of the patients $95.3 \%$ while other common findings wereLymphadenitis $81.3 \%$, Trismus 9.3\%, and Fistula 10.7\%.

\section{C-Abscess location:}

The spaces involved were identified according to clinical findings. The mostcommon space involved was buccal space abscess (48\%), dento-alveolar abscess (20.7\%), canine space abscess (18.7\%), Masseteric Space abscess (8\%), mental space abscess (4.7\%), sub-mandibular space abscess (4\%), palatal space abscess $(3.3 \%)$, and sublingual space abscess $(0.7 \%)$.

\section{D-Causative tooth:}

It was observed that majority of the infections were associated with mandibular teeth $(59.9 \%)$. The mandibular first molar $(27 \%)$ followed by mandibular first deciduous molar (17\%) and mandibular second deciduous molar and mandibular third molar (13\%) each. While in the maxilla (40.1\%), maxillary first molar (22\%) were most commonly involved teeth followed by the maxillary second premolar, maxillary central incisor, and maxillary first premolar $(17 \%)$ each.

\section{II- Bacteriology: A-Aerobic}

Figure (6) representing the aerobic bacterial results among studied patients. Among patients recruited in the study 133 patients $(88.7 \%)$ had aerobic bacterial infection. 109 patients had single bacterial isolate and 24 patients had multiple bacterial isolates. The most common isolated organism was Streptococcus Spp (89 patients representing $59.3 \%$ )

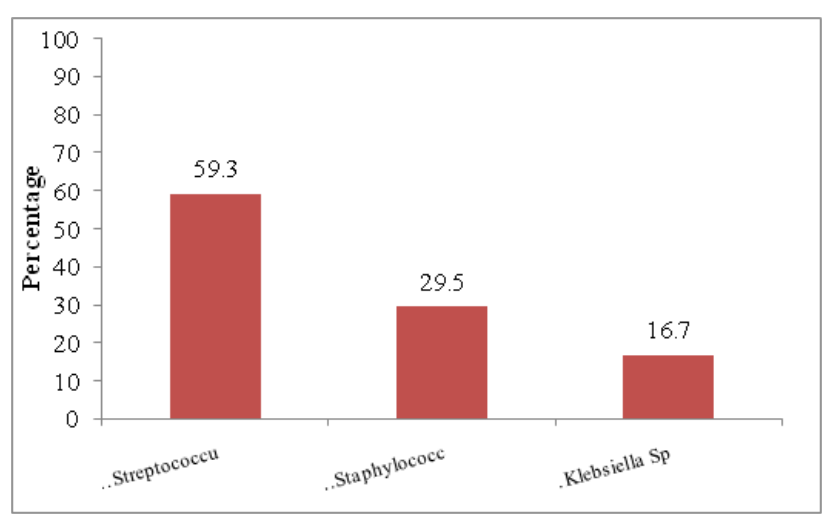

Fig. 6: Bar chart representing aerobic bacterial growth among studied patients.

\section{Antibiotic sensitivity pattern (aerobic)}

1. Staphyloccousspp (29.5\%) was isolated in 44 instances in which Ciprofloxacin and Amoxicillin/ clavulanic acid were the most sensitive drug $100 \%$ followed by Clindamycin $90.9 \%$ and Cefotaxime $77.27 \%$. The most resistant drugs were amoxicillin $86.37 \%$ and Metronidazole $100 \%$.

2. Streptococcus spp (59.3\%) was isolated in 89 instances in which Ciprofloxacin and Amoxicillin/ clavulanic acid were the most sensitive drug $100 \%$ followed by Clindamycin $91 \%$ and Cefotaxime $74.2 \%$. The most resistant drugs were amoxicillin $84.3 \%$ and Metronidazole $100 \%$.

3. Klebsiellaspp (16.7\%) was isolated in 25 instances in which Ciprofloxacin and Amoxicillin/clavulanic acid were the most sensitive drug $100 \%$ followed by Clindamycin $96 \%$ and Cefotaxime $72 \%$. The most resistant drugs were amoxicillin 84\% and Metronidazole 100\%.

Among the entire aerobically cultured bacteria, Ciprofloxacin and Amoxicillin/clavulanic acid were the 
most sensitive drug $100 \%$ followed by Clindamycin $90 \%$ and Cefotaxime $75 \%$. The leasteffective drug was amoxicillin $85 \%$.

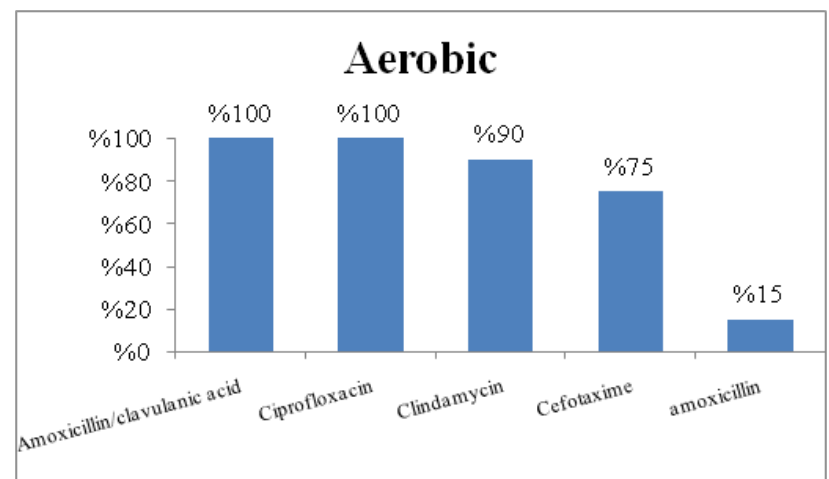

Fig. 7: Bar chart representing Antibiotic sensitivity pattern for aerobic overall

\section{B-Anaerobic \\ Pattern of isolated bacteria}

Figure (8) representing the anaerobic bacterial results among studied patients. Among patients recruited in the study 87 patients $(58.0 \%)$ had anaerobic bacterial infection. 71 patients $(47.3 \%)$ had single bacterial isolate and 16 patients $(10.7 \%)$ had multiple bacterial isolates. The most common isolated organism was Prevotella spp. (63 patients representing $42 \%$ ).

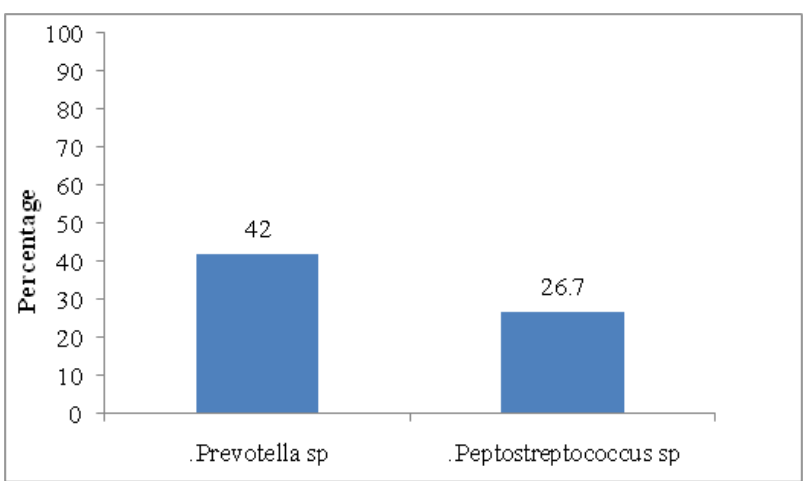

Fig. 8: Bar chart representing anaerobic bacterial growth

\section{Antibiotic sensitivity pattern (anaerobic)}

1. Peptostreptococcusspp $(26.7 \%)$ was isolated in 40 instances in which Metronidazole was the most sensitive drug (100\%) followed by Amoxicillin/clavulanic acid $(87.2 \%)$, Clindamycin $(84.6 \%)$, Cefotaxime and Ciprofloxacin $(71.8 \%)$ each. The most resistant drugs were amoxicillin (100\%).

2. Prevotellaspp (42\%) was isolated in 63 instances in which Metronidazole was the most sensitive drug (100\%) followed by Amoxicillin/clavulanic acid (90.5\%), Clindamycin and Ciprofloxacin (88.9\%) each, Cefotaxime
(79.4\%). The most resistant drugs were amoxicillin $(100 \%)$.

Among the entire anaerobically cultured bacteria, Metronidazole was the most sensitive drug (100\%) followed by Ciprofloxacin, Amoxicillin/clavulanic acid, Clindamycin $(90 \%)$ each and Cefotaxime $(80 \%)$. The leasteffective drug was amoxicillin (100\%).

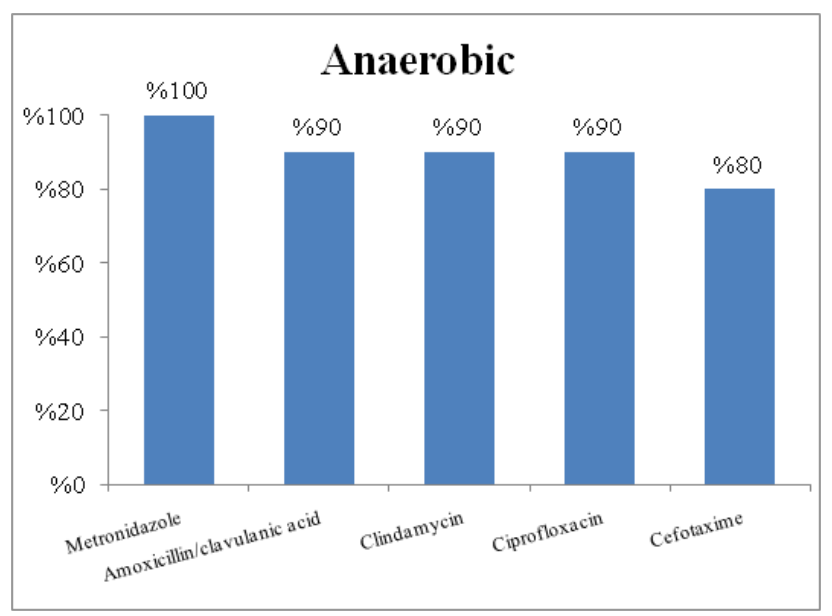

Fig. 9: Bar chart representing Antibiotic sensitivity pattern for anerobic overall.

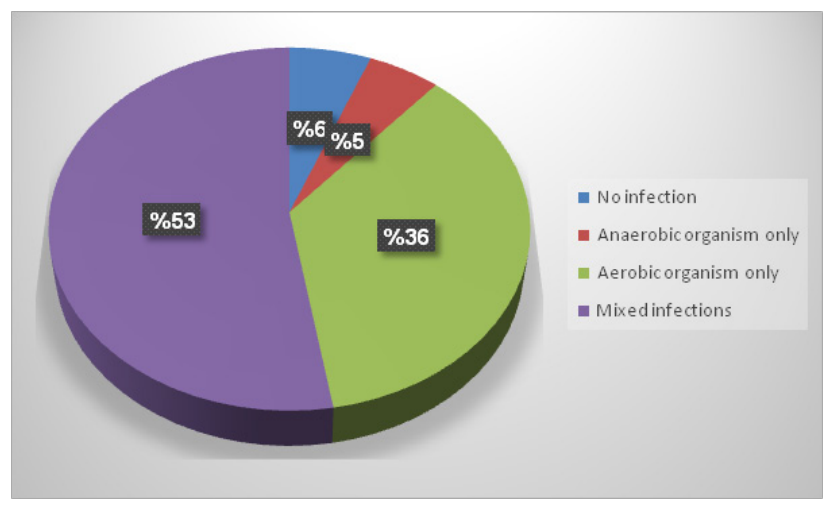

Fig. 10: pie chart representing bacterial isolates distribution among studied patients.

\section{DISCUSSION}

Most odontogenic infections arise as a sequel to pulp necrosis caused by caries or trauma. Periodontal infections, pericoronitis, trauma and surgery are other sources responsible for orofacial infections. Most of the odontogenic infections resolve with little consequences although occasionally complications may lead to more severe infection of the head and neck, particularly in immune-compromised or debilitated patients.

Kohli et al., (2009) who studied 80 patients with odontogenic infection noted that mandibular teeth were 
the most responsible for infection. The mandibular first molar was most commonly involved tooth followed by maxillary first molar and mandibular second molar. (Bahl et al., 2014) and (Fating et al., 2104) have reported in their studies that the mandibular third molar was the most common tooth to cause odontogenic infection representing $(31.70 \%)$ followed by the mandibular second molar and the mandibular first molar representing (17.07\%) and (12.21\%) respectively. In contrast Patankar et al., (2014) reported in his prospective study that up to $(66 \%)$ of the teeth responsible for odontogenic infection were maxillary teeth followed by the mandibular teeth $(44 \%)$.

In this study, it was observed that majority of the infections were associated with mandibular teeth (54\%). The mandibular first molar (18.6\%) and the maxillary first molar $(10.6 \%)$ were most commonly involved permanent teeth followed by the mandibular third molar (8.6\%) which was similar to the findings of the most of previously mentioned studies. The new finding is deciduous teeth presence as a part of the survey. The mandibular first deciduous molar (12\%) and the mandibular second deciduous molar $(10 \%)$ were most commonly involved deciduous teeth. The most common odontogenic infections observed were buccal space abscess $(47.3 \%)$ at the first place followed by dentoalveolar abscess $(21.3 \%)$ at the second place and canine space abscess at the third place (18\%). (Patankar et al., 2014) and (Bakathir et al., 2009) have also reported buccal space abscess at the first place $(41 \%)$ and $(96 \%)$, respectively.

The pathogenic bacteria of the oral cavity are complex and change with age, disease, and site. Studies noted that most of infections are $(65 \%$ to $70 \%)$ mixed aerobic and anaerobic, $(25 \%$ to $30 \%)$ anaerobic, and only (5\%) are aerobic. Most frequently isolated micro-organisms are aerobic streptococci, anaerobic streptococci, and bacteroides. The present study and (Lewis et al., 1986) isolated mixed micro-organisms in lower value (53\%) and (54\%) respectively. (Brook et al., 1991) and (Kohli et al., 2009) reported even lower values (44\%) and $(38 \%)$ respectively.

Only aerobic micro-organisms were isolated in (36\%) of the cases of the present study. This was similar to Kohli et al., (2009) who reported (35\%) aerobic infections but it was high in comparison with (Bahl et al., 2014)(25\%) and very high in comparison with (Brook et al., 1991) (6\%) and (Patankar et al., 2014) (8\%).

Only anaerobic micro-organisms were isolated in $(5 \%)$ of the cases of the present study. This was not similar to studies reported pure anaerobic infections. It was low in comparison with (Bahl et al., 2014)(15\%), (Patankar et al., 2014)(14\%), (Bakathir et al., 2009) (23\%), and (Kohli et al., 2009) (22.5\%), while very low in comparison with (Brook et al., 1991) (50\%) and (Lewis et al., 1986) (40\%).
Staphylococcus spp. including staphylococcus aureus was isolated in this study in total 44 cases $(29.5 \%)$. Similar percentage was reported by other investigators ${ }^{[2,5,8,11]}$ while (Fating et al., 2014), (Chunduri et al., 2012) and (Külekci et al., 1996) reported very low percentage (3.4\%), $(5.2 \%)$ and $(7.1 \%)$ respectively.

In this study, Streptococci spp. were isolated in 89(59.3\%) specimens. Only (Bahl et al., 2014) was similar reported isolation in $(45 \%)$ of the specimens. While, other investigators ${ }^{[6,9,10]}$ reported lower percentages. (Walia et al., 2014; Kohli et al., 2009) reported even lower values (15\%) and $(10 \%)$ respectively.

The isolation of Klebsiella in 25 cases $(16.7 \%)$ in this study is high when compared to other studies Kohli et al., (2009) reported (2.67\%), Patankar et al., (2014) reported (3\%), and Fating et al., (2014) reported (5\%). Only Walia et al., (2014) reported (10\%) which is similar to this study.

Prevotella spp. was isolated in 63(42\%) of the cases in this study. (Bahl et al., 2014;Chunduri et al., 2012; Külekci et al., 1996) reported (30\%), (25.7\%) and (25\%) of Prevotella spp. in their studies which is similar to this study. Other studies of (Fating et al., 2014) and (Walia et al., 2014) isolated very low percentage (1.7\%) and $(5 \%)$ respectively.

Gram-positive cocci like Peptostreptococcus spp. were also isolated in $40(26.7 \%)$ in this study that were similar to earlier studies of (Kohli et al., 2009)(29.46\%), (Bahl et al., 2014) (20\%). (Walia et al., 2014) reported lower value $(10 \%)$. Other studies reported high values (Patankar et al., 2014) (48\%), and (Fating et al., 2014) $(41.39 \%)$.

When a decision is made to prescribe antibiotics, severity of infection, normally present bacteria, antibiotic sensitivity test, patient's age and condition should be considered. It is the clinician's responsibility to choose the appropriate antibiotic therapy.

Among the aerobically cultured bacteria,Staphyloccous spp. including Staphyloccousaureus was sensitive to amoxicillin (13.63\%). Ciprofloxacin and Amoxicillin/ clavulanic acid were found (100\%) effective against all isolates ; while Clindamycin $(90.9 \%)$, and Cefotaxime (77.27\%). (Kohli et al., 2009) reported lower sensitivity value for amoxicillin (6.25\%) and similar value for ciprofloxacin $(100 \%)$. (Walia et al., 2014) reported higher sensitivity value for Amoxicillin and cefotaxime $(28.58 \%)$ and $(100 \%)$, respectively, and similar value for ciprofloxacin $(100 \%)$.

Streptococcus spp. was sensitive to amoxicillin (15.7\%). Ciprofloxacin and Amoxicillin/clavulanic acid were found $(100 \%)$ effective against all isolates while 
Clindamycin (91\%) and Cefotaxime (74.2\%). (Kohli et al., 2009) reported lower sensitivity value for ciprofloxacin $(85.71 \%)$ and similar value for amoxicillin (14.29\%). (Walia et al., 2014) reported higher sensitivity value for Amoxicillin and cefotaxime (75\%) and (100\%), respectively, and similar value for ciprofloxacin (100\%).

Klebsiella was sensitive to amoxicillin (16\%). Ciprofloxacin and Amoxicillin/clavulanic acid were found $(100 \%)$ effective against all isolates while Clindamycin (96\%), Cefotaxime (72\%), and Metronidazole (0\%) effective. Kohliet al., 2009) reported higher sensitivity value for amoxicillin (50\%) but lower value for Ciprofloxacin $(50 \%)$ and similar value for cefotaxime $(75 \%)$.

Among the entire aerobically cultured bacteria, Ciprofloxacin and Amoxicillin/clavulanic acid were the most sensitive drug $(100 \%)$ followed by Clindamycin $(90 \%)$ and Cefotaxime(75\%). The leasteffective drugs were amoxicillin (85\%). (Singh et al., 2014) reported high sensitivity value for amoxicillin and Cefotaxime $(78 \%)$ and (83\%), respectively, and similar value for Amoxicillin/ clavulanic acid (100\%); while lower value for ciprofloxacin (83\%). (Bahl et al., 2014) reported lower sensitivity value for ciprofloxacin and Amoxicillin/clavulanic acid (70\%) and $(90 \%)$, respectively while almost similar value for Clindamycin $(85 \%)$. (Fating et al., 2014) reported high sensitivity value for Cefotaxime and amoxicillin (95\%) and $(80 \%)$ respectively and similar value for ciprofloxacin and Clindamycin (95\%) each while lower value for Amoxicillin/clavulanic acid(80\%).

Among the anaerobically cultured bacteria, Peptostreptococcus spp. was $(0 \%)$ sensitive to amoxicillin. Ciprofloxacin and Cefotaxime were found (71.8\%) effective against all isolates while Clindamycin (84.6\%), Amoxicillin/clavulanic acid (87.2\%), and Metronidazole (100\%) effective. (Kohli et al., 2009) reported nearly similar sensitivity to Amoxicillin (5.9\%) while (Chunduri et al., 2012) reported very high sensitivity to Amoxicillin (91\%) and high values for Amoxicillin/ clavulanic acidandClindamycin $(100 \%)$ each.

Prevotella spp. was $(0 \%)$ sensitive to amoxicillin. Ciprofloxacin and Clindamycin were found (88.9\%) effective against all isolates while Amoxicillin/clavulanic acid (90.5\%), Cefotaxime (79.4\%), and Metronidazole (100\%) effective. (Kohli et al., 2009) reported nearly similar sensitivity to Cefotaxime $(75 \%)$ but high value for Amoxicillin (50\%) and low value for Ciprofloxacin (50\%). (Chunduri et al., 2012) reported very high sensitivity to Amoxicillin (78\%) and nearly similar values for Amoxicillin/clavulanic acid and Clindamycin (92\%) each.

Among the entire anaerobically cultured bacteria, Metronidazole was the most sensitive drug (100\%) followed by Ciprofloxacin, Amoxicillin/clavulanic acid, Clindamycin (90\%) each and Cefotaxime (80\%) The least effective drug was amoxicillin (100\%). Singh et al., (2014) reported high sensitivity values for amoxicillin and Amoxicillin/clavulanic acid (78\%) and $(100 \%)$, respectively while lower value for ciprofloxacin $(83 \%)$ and nearly similar value for Cefotaxime $(83 \%)$ (Bahl et al., 2014) reported lower sensitivity value for ciprofloxacin and Metronidazole (70\%) and (85\%) respectively while similar value for Amoxicillin/ clavulanic acid $(90 \%)$ and almost similar value for Clindamycin (85\%).

\section{CONCLUSION}

Based on the finding of this study, thefollowing conclusions were derived:

- The most common cause of odontogenic infection was found to be a mixture of aerobic and anaerobic bacteria $(53 \%)$ followed by aerobic bacteria $(36 \%)$.

- Pediatric patients were more prone to aerobic infection while adults patients were more prone to anaerobic infection.

- The micro-organisms isolated ranges from aerobic Streptococcus spp. (59.3\%) and Staphylococcus spp. $(29.5 \%)$ to anaerobic Peptostreptococcus spp. (26.7\%) and Prevotella spp. (42\%).

- For the aerobic isolates, Ciprofloxacin, Amoxicillin/ clavulanic acid and Clindamycin were the most sensitive drugs $(100 \%, 100 \%$ and $90 \%)$.

- For anaerobic isolates, Metronidazole was the most sensitive drug (100\%) followed by Ciprofloxacin, Amoxicillin/clavulanic acid and Clindamycin (90\%) each.

- For majority of isolates, Amoxicillin was found to be the most resistant.

\section{RECOMMENDATIONS}

- Similar studies in other governorates are recommended in order to provide overall a treatment modality with one antibiotic regimen on the whole Egyptian population.

- Ciprofloxacin, Amoxicillin/clavulanic acid and Clindamycinare recommended in odontogenic infections in combination with metronidazole for complete coverage of aerobic and anaerobic microorganisms.

- However, caution in the use of amoxicillin alone which is found to be resistant in spite of its wide spread use in odontogenic infection and as a prophylaxis in sub-acute endocarditis. 


\section{CONFLICT OF INTEREST}

There are no conflicts of interest.

\section{REFERENCES}

1. Abdulaziz A Bakathir, Khursheed F Moos, Ashraf F Ayoub, Jeremy Bagg (2009) Factors Contributing to the Spread of Odontogenic Infections : Sultan QaboosUniv Med J 9(3) : 296-304.

2. AmodPatankar, ArunDugal, Rajesh Kshirsagar, Hariram, Vikram Singh, Akshay Mishra (2014) Evaluation of microbial flora in orofacial space infections of odontogenic origin : Natl J MaxillofacSurg 5(2): 161-165.

3. Brook, I., Frazier EH, Gher ME. (1991) Aerobic and anaerobic microbiology of periapical abscess. Oral.Microbiol.Immunol. 6:123-5.

4. GüvenKülekçi, DilekİnançYaylali, HülyaKoçak, ÇetinKasapoglu, Osman ZekiGümrü (1996) Bacteriology of Dentoalveolar Abscesses in Patients Who Have Received Empirical Antibiotic Therapy :Clin Infect Dis 23(1) : 51-53.

5. Inderdeep Singh Walia, Rajiv M. Borle, D. Mehendiratta, Abhilasha O. Yadav (2014) Microbiology and Antibiotic Sensitivity of Head and Neck Space Infections of Odontogenic Origin: J. Maxillofac. Oral Surg. 13(1):16-21.
6. Lewis, M.A.O., MacFarlane, T.W., McGowan, O.A. (1986) Quantitative bacteriology of acute dentoalveolar abscesses. J. Med. Microb. 21:101-4.

7. Mamta Singh, Deepashri H. Kambalimath, K. C. Gupta (2014) Management of Odontogenic Space Infection with Microbiology Study: J. Maxillofac. Oral Surg. 13(2):133-139.

8. MunishKohli, AshaMathur, Monica Kohli3, SaifRaufSiddiqui (2009) In vitro evaluation of microbiological flora of orofacial infections : J Maxillofac Oral Surg 8(4): 329-333.

9. Nagendra S. Chunduri, KrishnaveniMadasu, Venkateswara R. Goteki, TanveerKarpe, Haranadha Reddy (2012) Evaluation of bacterial spectrum of orofacial infections and their antibiotic susceptibility : Ann. Maxillofac. Surg. 2(1): 46-50

10. Nitin Suresh Fating, D. Saikrishna, G. S. Vijay Kumar, Sujeeth Kumar Shetty, M. RaghavendraRao (2014) Detection of Bacterial Flora in Orofacial Space Infections and Their Antibiotic Sensitivity Profile : J Maxillofac Oral Surg 13(4):525-532.

11. RashiBahl, SumeetSandhu, Kanwardeep Singh, NilanchalSahai, Mohita Gupta Odontogenic infections (2014) Microbiology and management : ContempClin Dent 5(3): 307-311. 